\title{
Saturation of the ion drift instability in collisional complex plasmas by ion trapping
}

\author{
Victoria V. Yaroshenko $\odot$ \\ Institut für Materialphysik im Weltraum, Deutsches Zentrum für Luft- und Raumfahrt (DLR), 82234 Wessling, Germany
}

(Received 4 March 2021; accepted 1 June 2021; published 21 June 2021)

\begin{abstract}
Competition between ion trapping and collisional detrapping is considered to be an important issue in the stabilization of the ion flow instability. The saturation amplitudes in terms of the particle density are derived and compared to the experimental data.
\end{abstract}

DOI: 10.1103/PhysRevE.103.063209

\section{INTRODUCTION}

For some decades, many experimental and theoretical studies reported the excitation of dust density waves (DDWs) in various plasma and discharge conditions due to the socalled ion drift instability, and we refer to the recent review papers [1-3] and references therein. However, up to now all theoretical modelings of the ion drift instability have been restricted to the linear stage. At the same time, experiments and simulations of the wave excitation indicate that DDWs usually quickly approach a quasistationary state with finite amplitudes and thus are essentially nonlinear (see, e.g., Refs. [4-8]). In the nonlinear theory, the wave-particle interaction becomes an important factor. A wave with a finite amplitude can trap plasma particles moving with velocities close to the wave phase speed. This process modifies the initial (presumed) Maxwellian distribution function and respective dispersion relation, the instability growth rate or Landau damping, and enabling "steady-state" modes [9-11].

In this paper, we consider the saturation of the ion drift instability, incorporating the nonlinear effect of particle-wave interactions. We suggest a simple approach based on a close physical similarity between the current-driven ion-acoustic instability in conventional low-temperature plasmas and ion drift instability in complex plasmas. Thereby, we focus on the trapping processes for the lightest plasma species involved in the wave formation as discussed earlier in Refs. [12-17]. Hence, the plasma ions are assumed to be trapped by the potential structure of the DDWs. At the same time, the ion-neutral collisions can suppress the ion trapping. The equilibrium between these two counteracting processes defines the saturation amplitude.

The great advantage of a complex plasma is that the wave amplitudes in terms of particle density can be directly measured by optical methods and thus provide an opportunity to verify this mechanism. Below we use as input the amplitude measurements obtained in the nonlinear wave experiments of Refs. $[8,18,19]$. The experimental data are compared with the respective estimates of the theoretically derived saturation amplitudes. A favorable comparison of the measured amplitudes with theoretical predictions is a strong indication for the considered mechanism of saturation of the ion drift instability.

\section{SATURATION OF ELECTROSTATIC INSTABILITIES BY PARTICLE TRAPPING}

The linear theory provides only the instability conditions, specifying a frequency range or a wave-number width that can be excited, and results in unlimited, exponentially growing amplitudes. In reality, as the wave achieves a finite amplitude one needs to consider nonlinear processes that eventually lead to the saturation of the instability. There are many early theoretical and experimental studies of the interactions between an electron beam and the plasma waves which aim at understanding the nonlinear saturation of the electrostatic instabilities such as the plasma-beam (Buneman) and the ionacoustic instabilities (see, e.g., Refs. [11-17,20,21]). It has been widely accepted that the saturation of the waves occurs in the following manner: Initially, waves are unstable in a finite domain of the wave numbers. All these waves grow exponentially out of the thermal noise, but eventually the fastest growing mode necessarily adsorbs most of the energy so that it dominates the particle dynamics. A finite amplitude wave traps plasma particles moving with velocities close to the wave phase speed $v_{\mathrm{ph}}$, and the so-called resonant particles start to bounce back and forth in the potential structure of the wave with the bounce frequency $\omega_{B}=k(e \phi / m)^{1 / 2}\left[\right.$ here, $m=m_{e(i)}$ denotes the electron (ion) mass, $k$ is the wave number, and $\phi$ is the electric potential].

The nonlinear effect of the particle trapping becomes an essential factor whenever the wave frequency $\omega$ turns up to be significantly below the bounce frequency $\omega_{B}$. This introduces a threshold wave potential. Consider as an example ion-acoustic waves with a dispersion relation $\omega \simeq k \lambda_{\mathrm{De}} \omega_{p i}$, where $\omega_{p i}$ and $\lambda_{\mathrm{De}}$ are the ion plasma frequency and electron Debye length. It turns out that this mode can effectively trap electrons, already at the level of thermal fluctuations of the potential $\phi_{\mathrm{th}} \simeq \sqrt{T_{e} / \lambda_{\text {De }}}$ [12]. Indeed, the condition $\omega \ll \omega_{B e}$ is satisfied when the number of electrons in the electron Debye sphere obeys $\left(4 \pi n_{0 e} \lambda_{\mathrm{De}}^{3}\right)^{1 / 2} \ll m_{i} / m_{e}$, which is the case for many discharge plasmas [13].

The trapped resonant plasma particles modify the initial distribution function $f_{0 e(i)}$ tending to create a plateau in the vicinity of $v_{e(i)} \sim v_{\mathrm{ph}}$ with a width of $\Delta V_{\mathrm{tr}} \sim\left(e \phi / m_{e(i)}\right)^{1 / 2}$ in the electron (ion) velocity space. The formation of such 
a local plateau quenches the exponential growth of the wave amplitudes in the collisionless plasmas [11,16,17]. At the same time, particle collisions can remove plasma particles from the trapping region in velocity space. This reduces the trapping effect, the perturbed distribution function may become Maxwellian again, and the waves can grow to large values of $\phi[6,7,12,13]$.

In Ref. [12] it was considered that collisions can suppress trapping if the bounce frequency $\omega_{B}$ reaches the order of magnitude of the effective frequency for detrapping $v_{U}$. Thus, the condition $\omega_{B} \simeq v_{U}$ defines the saturation wave amplitude. In the case of ion-acoustic waves, the trapped electrons can become detrapped either due to Coulomb collisions or due to electron-neutral collisions. For the dominating Coulomb collisions, electrons have to diffuse through an angle $\sim\left(e \phi / T_{e}\right)^{1 / 2}$ which leads to the detrapping frequency $v_{U} \sim\left(e \phi / T_{e}\right) v_{C}$, where $v_{C}$ is the frequency of Coulomb collisions [13,15]. For the dominating electron-neutral collisions with the frequency $v_{e n}$, the detrapping frequency can be estimated as $v_{U} \sim$ $\left(\Delta V_{\text {tr } e} / V_{T e}\right)^{-2} v_{e n}$ [22]. Such scenarios of the ion-acoustic instability saturation have been discussed in experimental, numerical, and theoretical studies [6,7,13-15].

\section{EFFECT OF ION TRAPPING ON DDWs}

For standard dust-acoustic waves (DAWs) in typical experimental conditions, only ion trapping merits consideration, and the trapped ions induce an additional nonlinear frequency shift $\propto\left(e \phi / T_{i}\right)^{1 / 2}$ in the dispersion relation [23]. One can again formulate a condition of importance of the ion trapping effect as $\omega_{B i}=k\left(e \phi / m_{i}\right)^{1 / 2} \gg \omega$. Using a simplified dispersion relation for DAWs, $\omega \simeq k u_{d a}$, leads to $e \phi / T_{i} \gg \omega_{p d}^{2} / \omega_{p i}^{2}$. In this case, the dust-acoustic speed is given by $u_{d a}=\omega_{p d} \lambda \lambda_{D i}$ with $\omega_{p d}$ and $\lambda_{D i}$ being the dust plasma frequency and ion Debye length. For micron-sized particles employed in complex plasma research, the term $\omega_{p d}^{2} / \omega_{p i}^{2}$ is typically extremely small, $\omega_{p d}^{2} / \omega_{p i}^{2} \sim m_{i} Z_{d} / m_{d} \sim 10^{-9}-10^{-8}$, where $Z_{d}$ and $m_{d}$ refer to the particle charge number and mass, respectively. Therefore, even waves with a very small amplitude obeying $e \phi / T_{i} \ll 1$ can trap ions. Qualitatively similar results can be expected for all dust density waves propagating in variety of experimental conditions.

Assuming the importance of ion trapping for DDWs, one has to keep in mind that the ion distribution function (IDF) in the presence of electric fields and ion-neutral collisions can deviate from the shifted Maxwellian distribution [24,25]. The function $f_{0 i}$ has a slower exponential decay in the direction of the ion flow ( $x$ axis) than a respective Maxwellian distribution and becomes broader, with an effective "temperature" increase with the thermal ion Mach number $\propto T_{i}\left(1+M_{i}^{2}\right)$. Moreover, the IDF maximum is not shifted towards ion velocities $V_{x} / V_{T i} \sim M_{i}$, but occurs at lower velocities (see, e.g., in Fig. 1 in Ref. [25], $V_{x} / V_{T i} \lesssim 2$ for $M_{i} \lesssim 9$ ). Here, the thermal Mach number stands for $M_{i}=u_{0} / V_{T i}$ with $u_{0}$ and $V_{T i}$ being the ion drift and thermal velocities, respectively.

In the case of the dust density waves, the phase velocity $v_{\mathrm{ph}}$ is much lower then the ion thermal velocity, $v_{\mathrm{ph}} \ll V_{T i}$, and hence the ions will always be trapped in the bulk of their distribution. The resonant interaction covers velocity space $v_{\mathrm{ph}}-$ $\Delta V_{\mathrm{tr}} / 2<V_{x}<v_{\mathrm{ph}}+\Delta V_{\mathrm{tr}} / 2, \quad$ where $\quad \Delta V_{\mathrm{tr}}=2\left(e \phi / m_{i}\right)^{1 / 2}$

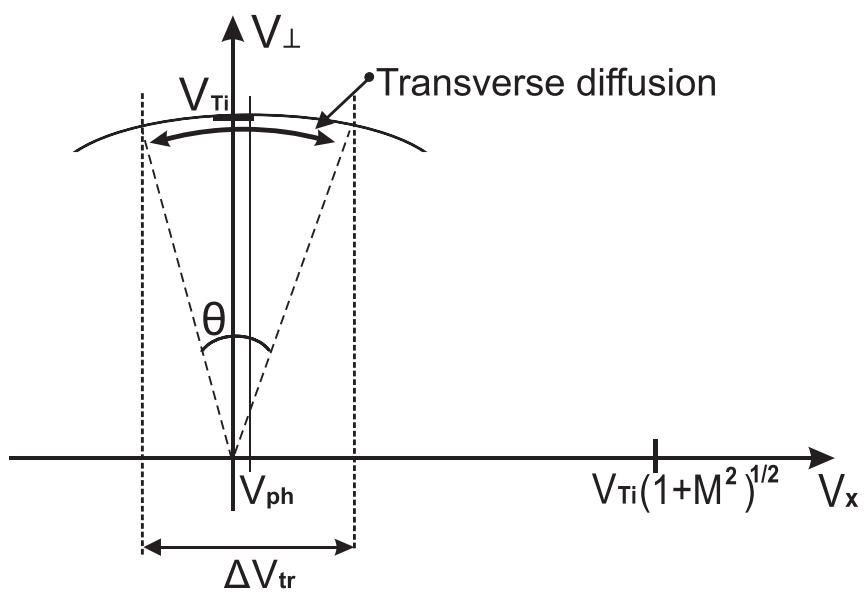

FIG. 1. Collisional detrapping of resonant ions in the bulk of the ion distribution in the case of DDWs. The figure has been respectively adapted from Ref. [22].

denotes the full ion trapping width. For particles trapped in the bulk of their distribution, only transverse diffusion may lead to collisional detrapping and we estimate the characteristic timescale for the ion detrapping in the same way as the electron detrapping time in the case of ion-acoustic waves [22]. Note that in order to correctly account for pitch angle scattering (transverse diffusion) one needs an additional velocity space direction $V_{\perp}$, perpendicular to the direction of the wave propagation, as schematically shown in Fig. 1. It is plausible to assume that in this direction the IDF is Maxwellian. In complex plasmas, the charged species collide mostly with neutral atoms, and the ion-neutral collisions represent the main effective mechanism which can provide for ion detrapping. The average ion scattering angle $\theta \sim \Delta V_{\mathrm{tr}} / \mathrm{V}_{T i}$ (see Fig. 1) gives the characteristic time for the ion detrapping by transverse collisional diffusion $v_{U}^{-1} \simeq \theta^{2} / v_{\text {in }} \sim \Delta V_{\text {tr }}^{2} /\left(\mathrm{V}_{T i}^{2} v_{i n}\right)$. In the latter expression the ion-neutral collision frequency $v_{\text {in }}$ has to be calculated at the ion velocity $V_{\perp} \simeq V_{T i}$.

Following the idea of the nonlinear saturation of the ion-acoustic instability [12], we assume that the ion drift instability of DDWs can be suppressed when the ion bounce period $\sim \omega_{B i}^{-1}$ reaches the order of magnitude of the ion detrapping time $v_{U}^{-1}$. This provides the equation which determines the saturation potential $\phi_{s}$,

$$
\frac{1}{k \sqrt{\left(e \phi_{s} / m_{i}\right)}} \simeq \frac{4 e \phi_{s}}{T_{i} \nu_{i n}} .
$$

For the dimensionless quantity $\varphi=e \phi_{s} / T_{i}$ the solution reads as

$$
\varphi \simeq \sqrt[3]{\frac{v_{i n}^{2}}{16 k^{2} V_{T i}^{2}}}
$$

Increasing the gas pressure $p_{n}$ yields a higher saturation potential $\varphi$ for the same $k$. Physically, this means that the growth of the ion-neutral collisions could reduce ion trapping, so that a wave with the same wave number $k$ would grow to a larger $\varphi$. 
In order to give an idea of the values of the saturation level (2), we estimate $\varphi$ in the parameter space of some DDW experiments performed in argon gas. The different normalized potentials are computed as $\varphi \sim 0.6$ for the most unstable wave number $k \sim 30 \mathrm{~cm}^{-1}$ at pressure $p_{n}=12 \mathrm{~Pa}$ in Ref. [26]; $\varphi \sim 1$ for $k \sim 20-30 \mathrm{~cm}^{-1}$ at $p_{n}=16.5 \mathrm{~Pa}$ [27], and $\varphi \sim$ 1.5 for $k \sim 45 \mathrm{~cm}^{-1}$ at $p_{n}=50 \mathrm{~Pa}$ [8]. The attained values $\varphi=e \phi_{s} / T_{i} \sim 1$ are consistent with the order of magnitude estimates of $\varphi$ obtained in the particle-in-cell (PIC) simulations of the ion flow instability in collisional plasmas [6]. However, in order to apply the estimates of $\varphi$ to experimental data, one needs to determine how the electrostatic potential (2) is related to the wave amplitudes in terms of the particle density that can be directly measured. The standard procedure includes analysis of the wave video images and calculation of space-time diagrams, where the brightness of the image is proportional to the local particle density $n_{d}$. For example, one can define the DDW amplitudes as dust density variations $\delta n_{d}$ with respect to their mean (background) value $n_{0 d}$, i.e., $\delta n_{d} / n_{d 0}=\left|n_{d}-n_{d 0}\right| / n_{d 0}$.

Explicit expressions for $n_{d}(\varphi)$ can be obtained only for the most simple cases. For the waves with small finite amplitudes $\delta n_{d} / n_{0 d}<1$, one can expect that the relation between $\delta n_{d} / n_{0 d}$ and the associated potential $\phi_{s}$ is close to that given by the linearized fluid-dynamical dust momentum and continuity equations, viz.,

$$
\frac{\delta n_{d}}{n_{0 d}} \simeq\left|-\frac{e \phi_{s} Z_{d} k^{2}}{\omega\left(\omega+i \nu_{d n}\right) m_{d}}\right|
$$

where $v_{d n}$ denotes the dust-neutral collision frequency. Inserting the potential $\varphi=e \phi_{s} / T_{i}$ in the form of (2) gives the saturation level of the particle density as

$$
\frac{\delta n_{d}}{n_{0 d}} \simeq \varphi \frac{k^{2} T_{i} Z_{d}}{m_{d} \sqrt{\left(\omega_{r}^{2}+\gamma^{2}\right)\left[\omega_{r}^{2}+\left(\gamma+v_{d n}\right)^{2}\right]}} .
$$

Here, $\omega_{r}$ and $\gamma$ refer to the real and imaginary part of the wave frequency.

Another possibility for evaluating $n_{d}(\varphi)$ is to consider the initial nonlinear momentum and continuity equations for the dust component under the assumption of cold particles and neglecting the dust-neutral collisions. The latter requires that the nonlinear wave frequency obeys $\omega \gg v_{d n}$. In this case, one works in a frame which follows the nonlinear structure, and all quantities depend on the space variable $x$ and time variable $t$ through a combination of $\varsigma=x-V t$, where $V$ is the velocity of the nonlinear wave. The set of the dust continuity and momentum equation can be integrated with vanishing perturbations at $\zeta \rightarrow \infty$ in a standard way (see, e.g., Ref. [28]), yielding

$$
\frac{n_{d}}{n_{0 d}}=V\left[V^{2}+2 e Z_{d} \phi / m_{d}\right]^{-1 / 2} .
$$

Assuming $V \simeq \omega / k$ and $\phi=\phi_{s}$ leads to the saturation amplitude

$$
\frac{\delta n_{d}}{n_{0 d}}=1-\frac{1}{\sqrt{1+2 \varphi k^{2} T_{i} Z_{d} /\left(m_{d} \omega^{2}\right)}} .
$$

Inserting the complex plasma parameters and wave frequencies in the appropriate Eq. (4) or (6) provides the saturation amplitude in terms of the particle density for a given wave number. The validity of the model for saturation of the ion flow instability can be then verified by comparison of the obtained theoretical values of $\delta n_{d} / n_{0 d}$ to the respective experimentally determined wave amplitudes.

\section{COMPARISON WITH EXPERIMENTAL DATA}

Successful studies of the nonlinear dust density waves performed in the ground-based experiments have been recently reported in Refs. [8,18,19]. Microparticles of $4.8 \mu \mathrm{m}$ diameter were electrically confined using a glass box in the argon rf plasma at pressures 372-420 mTorr. The plane-wave excitation occurred due to a downward ion flow at $p_{n} \lesssim 420$ $\mathrm{mTorr}$ and the wave propagation was one dimensional. Video images of the self-excited DDWs were used for calculation of the space-time diagram, power spectrum, and the amplitude detection. The analysis at different gas pressures thus resulted in the measured wave amplitudes (in terms of the particle density), wave number, frequency, and growth rate of the most unstable DDW. For experimental details, a description of the analysis methods, as well as estimates of the plasma and particle parameters, we refer to original papers $[8,18,19]$.

While previous studies of the nonlinear waves in Ref. [8] mostly focused on the wave form and its interpretation as an exact solution of the Korteweg-de Vries equation, in this paper, we analyze the wave amplitudes at the final stage of the ion flow instability. Being close to the linear perturbations in the upper part of the particle cloud, the waves achieve the highest amplitudes in the lower part. Hence, for our analysis we select two types of data associated with the most downstream parts of the cloud [so-called regions of interest ROI-4 and ROI-5, identified in Fig. 2(a) of Ref. [8]]. Moreover, in order to avoid a strong effect of the higher harmonic generation, that grows with decreasing pressure, we restrict our analysis to the narrow range of gas pressures in the vicinity of the instability threshold and consider $p_{n} \sim 404-416$ mTorr.

First, we estimate the theoretical saturation amplitudes of the DDWs due to the ion flow instability in the experimental parameter space. For this purpose, we employ a set of the complex plasma parameters in the argon discharge listed in Table I of Ref. [18]. Moreover, we use the data of Table I of Ref. [8], where one finds the experimentally measured values of the wave frequencies $f=\omega / 2 \pi$ and relative damping rate $v_{d n} / 2 \pi f$ for a given gas pressure. In the regions of interest ROI-4 and ROI-5 $v_{d n} / 2 \pi f \sim 0.7-0.8$ and thus for evaluation of the saturation amplitudes one can use the expression (4) accounting for the particle-neutral collisions. Furthermore, at the considered high gas pressures the maximum of the instability growth rate $\gamma$ remains much lower than the respective wave frequency $2 \pi f$, and $\gamma$ can be safely neglected in our further calculations. Here, the $\gamma$ estimate has been computed as $\gamma \sim\left(k_{i} V_{g}\right)$ using the highest spatial growth rate $k_{i} \sim 7 \mathrm{~cm}^{-1}$ and the group velocity $V_{g} \sim 3 \mathrm{~cm} / \mathrm{s}$ found in the plots in Figs. 6 and 7 of Ref. [19]. Finally, we take into account that the nonlinear waves are excited at the same wave number $k \sim 45 \mathrm{~cm}^{-1}$ independently of the gas pressure.

It is important to notice that even weak nonlinearity of the system modifies the wave dispersion relation. The latter becomes dependent on a finite wave amplitude, and thus the 

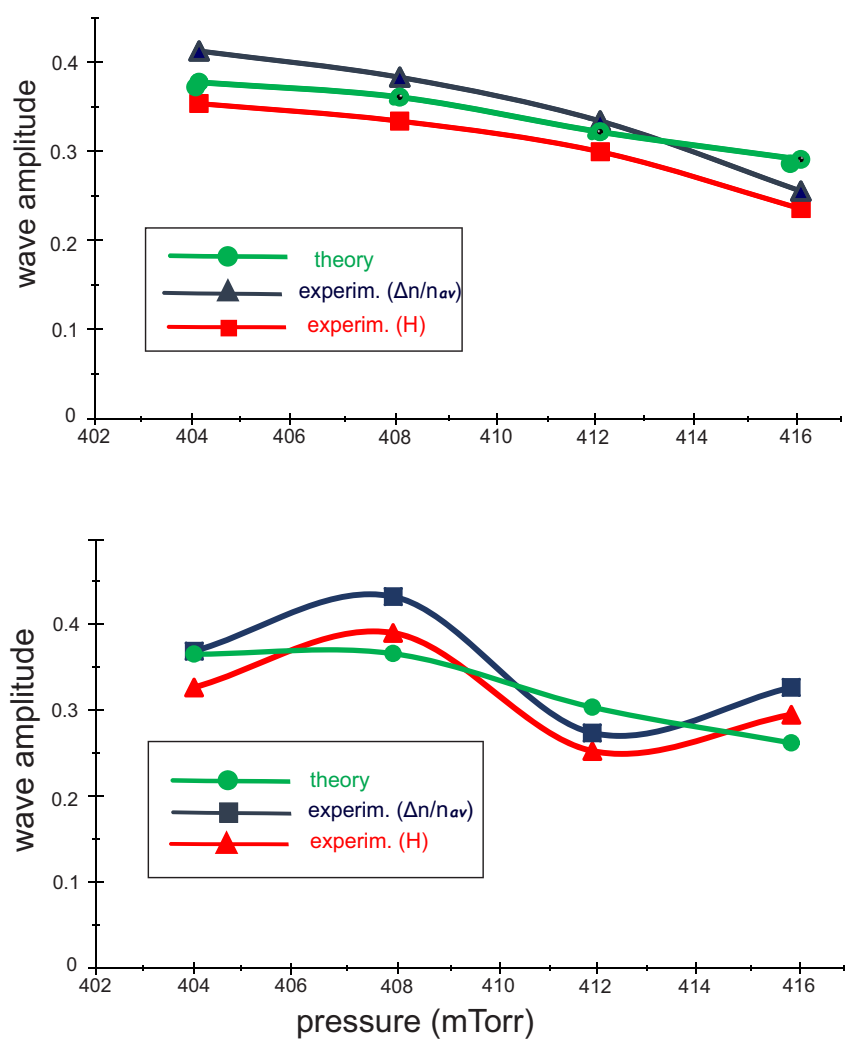

FIG. 2. Theoretical saturation level (4) and experimental estimates of the wave amplitude $\delta n_{d} / n_{0 d}$ defined through values of $\Delta n / n_{\text {av }}$ and $H$ of Table I in Ref. [8] in two different parts of the particle cloud, ROI-4 (upper panel) and ROI-5 (lower panel), at various gas pressures. The regions ROI-4 and ROI-5 are identified in Fig. 2 of Ref. [8].

measured frequencies $f$ in Table I of Ref. [8] cannot be directly associated with the linear frequencies $\omega_{r}$, involved in Eq. (4). In other words, we cannot use the simple relation $\omega_{r}=2 \pi f$. To derive the nonlinear dispersion relation, we apply the approach considered previously for DAWs in collisionless plasmas [23].

We assume that the measured wave frequency can be presented in the form $f \simeq f_{L}+\Delta f$, where $f_{L}=\omega_{r} / 2 \pi$ denotes the linear wave frequency. The nonlinear frequency shift $\Delta f$ that appears due to the trapped ions can be estimated as [23]

$$
\Delta f \simeq-\left.\alpha \frac{\omega_{p i}^{2}}{2 \pi k^{2}} \Delta V_{\operatorname{tr}}\left[\frac{\partial \epsilon_{L}\left(\omega_{r}\right)}{\partial \omega}\right]^{-1} \frac{\partial^{2}\left(f_{0 i} / n_{0 i}\right)}{\partial V_{x}^{2}}\right|_{\omega_{r} / k},
$$

where $n_{0 i}$ refers to the background ion density and $\epsilon_{L}$ is the linear dielectric function. For the propagating DDWs $\left(\omega_{r}>\right.$ $\gamma$ ), one can use as a first-order approximation

$$
\frac{\partial \epsilon_{L}\left(\omega_{r}\right)}{\partial \omega} \simeq \frac{2 \omega_{p d}^{2} \omega_{r}}{\left(\omega_{r}^{2}+v_{d n}^{2}\right)^{2}}
$$

In the considered experiments $M_{i} \lesssim 2$ [18], and the ion distribution function along the ion flow direction can be approximated by the Maxwellian IDF with an increased width

$$
\begin{aligned}
& \sim V_{T i}\left(1+M_{i}^{2}\right)^{1 / 2} . \text { For } \omega_{r} / k \ll V_{T i} \text { this yields } \\
& \qquad \frac{\partial^{2}\left(f_{0 i} / n_{0 i}\right)}{\partial V_{x}^{2}} \simeq-\frac{1}{\sqrt{2 \pi} V_{T}^{3}\left(1+M_{i}^{2}\right)^{3 / 2}} .
\end{aligned}
$$

Inserting the expressions (8) and (9) into (7) gives a positive frequency shift $\Delta f \propto \sqrt{\varphi}$ and provides the following relation between the frequencies $f$ and $\omega_{r}$,

$$
2 \pi f \simeq \omega_{r}\left(1+\frac{\alpha\left(\omega_{r}^{2}+v_{d n}^{2}\right)^{2} \sqrt{\varphi}}{\sqrt{2 \pi} k^{2} u_{d a}^{2} \omega_{r}^{2}\left(1+M_{i}^{2}\right)^{3 / 2}}\right) .
$$

Here, the numerical coefficient $\alpha$ depends on the distribution of the trapped resonant ions [29]. If the wave is driven slowly with respect to the bounce timescale by an external force up to a finite amplitude, it evolves to a so-called adiabatic distribution of resonant particles and $\alpha=0.544$. For the above-considered plasma parameters, the wave number $k \sim 45 \mathrm{~cm}^{-1}$, and the potential $\varphi \sim 1$, one finds the following ordering of the characteristic timescales: The bounce time $\omega_{B i}^{-1} \sim 10^{-6}-10^{-5} \mathrm{~s}$ is much smaller than the timescale of the wave growth due to the ion flow instability which is $\gamma^{-1} \sim$ $1 /\left(k_{i} V_{g}\right) \gtrsim 10^{-2}-10^{-1} \mathrm{~s}$. Therefore the adiabatic distribution of the resonant ions is well suited for our estimates.

Using the saturation potential (2) and solving (10) numerically for a given $f$ at a certain pressure yields the respective wave frequency $\omega_{r}$, that should be inserted into Eq. (4) that determines the amplitude in terms of particle density. In our calculations, we consider the average wave number $k \sim$ $45 \mathrm{~cm}^{-1}$, the electric field $E \sim 15 \mathrm{~V} / \mathrm{cm}$, and the ion density $n_{0 i} \sim 6 \times 10^{8} \mathrm{~cm}^{-1}$, and all other parameters are specified in Table I of Ref. [18]. Figure 2 illustrates obtained estimates for $\delta n_{d} / n_{0 d}$ as a function of gas pressure for two different regions ROI4 and ROI-5. The model indicates a weak decrease of the saturation amplitude $\delta n_{d} / n_{0 d}$ with gas pressure in both regions of interests.

Furthermore, we compare the obtained values of $\delta n_{d} / n_{0 d}$ with respective amplitude measurements given in Table I of Ref. [8]. In the experiments, the peak-to-peak amplitudes $\Delta n$ were normalized in two different ways, either to the average particle density $n_{\text {av }}$ or to the minimum observed density $H=$ $\Delta n / n_{\text {min }}$. Adapting the measured values of $\Delta n / n_{\text {av }}$ and $H$ to the introduced $\delta n_{d} / n_{0 d}$ leads either to $\delta n_{d} / n_{0 d} \simeq \Delta n / 2 n_{\mathrm{av}}$ or to $\delta n_{d} / n_{0 d} \simeq H /(2+H)$. For a given gas pressure, we thus have two measured values of $\delta n_{d} / n_{0 d}$. In Fig. 2, we superimpose both experimental amplitudes $\left(\delta n_{d} / n_{0 d}\right)$ on the respective theoretical values. It can be clearly seen that the theoretical values match well to the trends in the experimental data, revealing quite good qualitative and quantitative agreement in the considered range of gas pressure for both regions of interests and the ion flow instability saturates at the level $\delta n_{d} / n_{0 d} \sim 0.25-0.4$.

Before closing this section, it is worth speculating about the saturation level at significantly lower pressures, when the DDW resembles a standard dust-acoustic mode. Assuming $\omega>v_{d n}, \gamma$, the saturation amplitude follows from Eq. (6) as $\delta n_{d} / n_{0 d} \simeq 1-\left[1+2\left(1+k^{2} \lambda_{D i}^{2}\right) \varphi / p\right]^{-1 / 2}$, where $p$ refers to the Havnes parameter $p=Z_{d} n_{0 d} / n_{0 i}$. Considering as an example the parameter space of the earlier mentioned wave experiment at $p_{n}=16.5 \mathrm{~Pa}$ (124 mTorr) [27] and assuming 
$p \sim 1$ gives a minimum saturation amplitude $\delta n_{d} / n_{0 d} \sim 0.5$. The obtained estimate of $\delta n_{d} / n_{0 d} \sim 0.5$ seems to be consistent with variations of the wave image intensity observed in the bulk of the particle cloud as shown in Fig. 3(a) of Ref. [27].

\section{CONCLUSIONS}

In this paper, we discuss a possible mechanism responsible for the saturation of the ion flow instability in complex plasmas based on ion trapping that is modified by ion-neutral collisions. The idea is based on previous studies of the nonlinear saturation of the ion-acoustic instability in conventional plasmas [12,13] and the close similarity between ion-acoustic and dust-acoustic waves. The important feature of the described approach is a single wave model that is based on the fact that already at the linear stage, the fastest growing mode adsorbs most of the energy as it grows and thus strongly dominates the particle-wave interaction. Furthermore, it is assumed that the equilibrium between the resonant ion trapping by the wave of a finite amplitude and detrapping by ion-neutral collisions is a key process in the nonlinear saturation of the ion flow instability.

In such a scenario, the predicted saturation potentials in the typical experiments $\varphi=e \phi_{s} / T_{i} \sim 1$ are consistent with the order of magnitude of the respective estimates obtained in PIC simulations [6]. In order to apply the results to specific wave experiments, the wave amplitudes have been recalculated as a function of the particle density. Estimations of the saturation level of the particle density have been performed in the parameter space of the wave experiments $[8,18,19]$, based on the linearized relation (4) that was correspondingly modified in order to account for the nonlinear contribution to the experimentally measured frequency. Theory leads to the maximal amplitudes $\delta n_{d} / n_{0 d} \sim 0.3-0.4$ slightly decreasing with the gas pressure (Fig. 2). The theoretically derived values and the overall trend are fully consistent with the amplitude measurements of the self-excited DDWs at the bottom of a dust cloud as described in Ref. [8]. The estimated saturation levels $\delta n_{d} / n_{0 d} \gtrsim 0.5$ expected at lower pressures in the condition of the experiment [27] are also supported by the measurements.

The agreement between theory and experiments within the considered plasma conditions seems to be surprisingly good, keeping in mind some of the simplified assumptions such as fixed particle charge and density, constant electric field and wave number, an approximated ion distribution function, etc. We remark also that we have left aside the generation of higher harmonics. At the same time, a closer look at Fig. 2 indicates that the best fit is observed in the ROI-4 part of the cloud, where the deviations between the theoretical and the experimental amplitudes are $\sim 10 \%$, while in the most downstream ROI-5 part they can reach up to $20 \%$. The growing discrepancies in ROI-5 can be explained by the generation of higher harmonics. In fact, the analysis of the experimental data in Ref. [19] revealed the appearance of Fourier harmonics of the fundamental mode in the Fourier spectrum of the DDWs at the bottom of the dust cloud (ROI-5). The increasing role of the higher harmonics might additionally influence the dispersion relation (see, e.g., Ref. [23]) and thus the theoretical estimates of $\delta n_{d} / n_{0 d}$.

This theoretical consideration of the saturation of the ion flow instability, which establishes an agreement of the measured amplitudes with theoretical predictions, indicates that competition between ion trapping and collisional detrapping can be important for the saturation of the ion drift instability. However, more experiments are required to provide a further test of the presented saturation mechanism over a wide range of discharge conditions. At the same time, the experimental evidence highlights the need for a more complete theory, accounting for both nonlinear effects, the particle-wave interaction, and harmonic generation, which appears still to be lacking.

\section{ACKNOWLEDGMENT}

The author thanks Dr. Mierk Schwabe for a careful reading of the manuscript.
[1] R. L. Merlino, 25 years of dust acoustic waves, J. Plasma Phys. 80, 773 (2014).

[2] P. K. Shukla and B. Eliasson, Fundamentals of dust-plasma interactions, Rev. Mod. Phys. 81, 25 (2009).

[3] V. E. Fortov, A. V. Ivlev, S. A. Khrapak, A. G. Khrapak, and G. E. Morfill, Complex (dusty) plasmas: Current status, open issues, perspectives, Phys. Rep. 421, 1 (2005).

[4] R. L. Merlino, R. Heinrich, S.-H. Kim, and J. K. Meyer, Dusty plasmas: Experiments on nonlinear dust acoustic waves, shocks and structures, Plasma Phys. Controlled Fusion 54, 124014 (2012).

[5] R. L. Merlino, R. Heinrich, S.-H. Hyun, and J. K. Meyer, Nonlinear dust acoustic waves and shocks, Phys. Plasmas 19, 057301 (2012).

[6] K. Quest, M. Rosenberg, and B. Kercher, Simulations of the ion-dust streaming instability with non-Maxwellian ions, J. Plasma Phys. 83, 905830612 (2017).
[7] D. Winske and M. Rosenberg, Nonlinear development of the dust acoustic instability in a collisional dusty plasma, IEEE Trans. Plasma Sci. 26, 92 (1998).

[8] B. Liu, J. Goree, T. M. Flanagan, A. Sen, S. K. Tiwari, G. Ganguli, and C. Crabtree, Experimental observation of cnoidal waveform of nonlinear dust acoustic waves, Phys. Plasmas 25, 113701 (2018).

[9] B. Bernstein, J. M. Greene, and M. D. Kruskal, Exact nonlinear plasma oscillations, Phys. Rev. 108, 546 (1957).

[10] T. M. O’Neil, Collisionless damping of nonlinear plasma oscillations, Phys. Fluids 8, 2255 (1965).

[11] R. Z. Sagdeev, and A. A. Galeev, Nonlinear Plasma Theory Rev. and edited by T. M. O'Neil and D. L. Book (W. A. Benjamin, New York, 1969).

[12] K. Nishikawa and C.-S. Wu, Effect of Electron Trapping on the Ion-Wave Instability, Phys. Rev. Lett. 23, 1020 (1969). 
[13] M. Yamada and M. Raether, Saturation of the Ion-Acoustic Instability in a Weakly Ionized Plasma, Phys. Rev. Lett. 32, 99 (1974).

[14] M. Yamada and M. Raether, Evolution of the ion-acoustic instability in a direct-current discharge plasma, Phys. Fluids 18, 361 (1975).

[15] N. W. Albright, Stabilization of ion acoustic waves by electron trapping, Phys. Fluids 17, 206 (1974).

[16] R. L. Dewar, Saturation of kinetic plasma instabilities by particle trapping, Phys. Fluids 16, 431 (1973).

[17] T. M. O'Neil, J. H. Winfrey, and J. H. Malmberg, Nonlinear interaction of a small cold beam and a plasma, Phys. Fluids 14, 1204 (1971).

[18] T. M. Flanagan and J. Goree, Observation of the spatial growth of self-excited dust-density waves, Phys. Plasmas 17, 123702 (2010).

[19] T. M. Flanagan and J. Goree, Development of nonlinearity in a growing self-excited dust-density wave, Phys. Plasmas 18, 013705 (2011).

[20] D. Winske, S. P. Gary, M. E. Jones, M. Rosenberg, V. W. Chow, and D. A. Mendis, Ion heating in a dusty plasma due to the dust/ion acoustic instability, Geophys. Res. Lett. 22, 2069 (1995).

[21] H. Fu and W. Scales, Nonlinear Evolution of the dust acoustic instability in artificially created dusty space plasmas, IEEE Trans. Plasma Sci. 40, 1223 (2012).
[22] R. L. Berger, S. Brunner, T. Chapman, L. Divol, C. H. Still, and E. J. Valeo, Electron and ion kinetic effects on non-linearly driven electron plasma and ion acoustic waves, Phys. Plasmas 20, 032107 (2013).

[23] V. V. Yaroshenko, Nonlinear dispersion relation for dustacoustic waves in complex plasmas, Phys. Rev. E 102, 023201 (2020).

[24] M. Lampe, T. B. Rocker, G. Joyce, S. K. Zhdanov, A. V. Ivlev, and G. E. Morfill, Ion distribution function in a plasma with uniform electric field, Phys. Plasmas 19, 113703 (2012).

[25] H. Kaelert, Ion-dust streaming instability with non-Maxwellian ions, Phys. Plasmas 22, 073703 (2015).

[26] V. V. Yaroshenko, B. M. Annaratone, S. A. Khrapak, H. M. Thomas, G. E. Morfill, V. E. Fortov, A. M. Lipaev, V. I. Molotkov, O. F. Petrov, A. I. Ivanov, and M. V. Turin, Electrostatic modes in collisional complex plasmas under microgravity conditions, Phys. Rev. E 69, 066401 (2004).

[27] V. Nosenko, S. K. Zhdanov, S.-H. Kim, J. Heinrich, R. L. Merlino, and G. E. Morfill, Measurements of the power spectrum and dispersion relation of self-excited dust acoustic waves, Europhys. Lett. 88, 65001 (2009).

[28] F. Verheest, Waves and instabilities in dusty space plasmas, Space Sci. Rev. 77, 267 (1996).

[29] R. L. Dewar, Frequency shift due to trapped particles, Phys. Fluids 15, 712 (1972). 